\title{
"Nous pouvons donner une seconde chance à beaucoup de patients»
}

\section{Daniel Lüthi}

Journaliste indépendant et photographe, conseiller médiatique, Berne

Dans le bureau, le regard est d'abord attiré par une photo de montagnes. Sur la photo en noir et blanc, on aperçoit des vaches, des enfants et une cabane alpine. «La cabane de ma mère» commente Jürg Steiger, «Falein près de Filisur - un lieu important pour notre famille. «Nous allons vers l'ordinateur et regardons les clichés de famille pris lors de jours de fête, des gens gais autour d'une grande table. «Une tribu soudée», déclare Jürg Steiger.

\section{L’esprit de médecin de famille}

Il est rare que la rencontre avec un médecin soit si rapidement aussi une rencontre avec sa vie privée. Généralement, les spécialistes renommés veulent parler avant tout, voire uniquement, de leur univers professionnel. Ce professeur parle de ce qui lui vient à l'esprit, par exemple du chien qui l'accompagne dans son footing matinal et de ses deux filles adultes qui vivent encore à

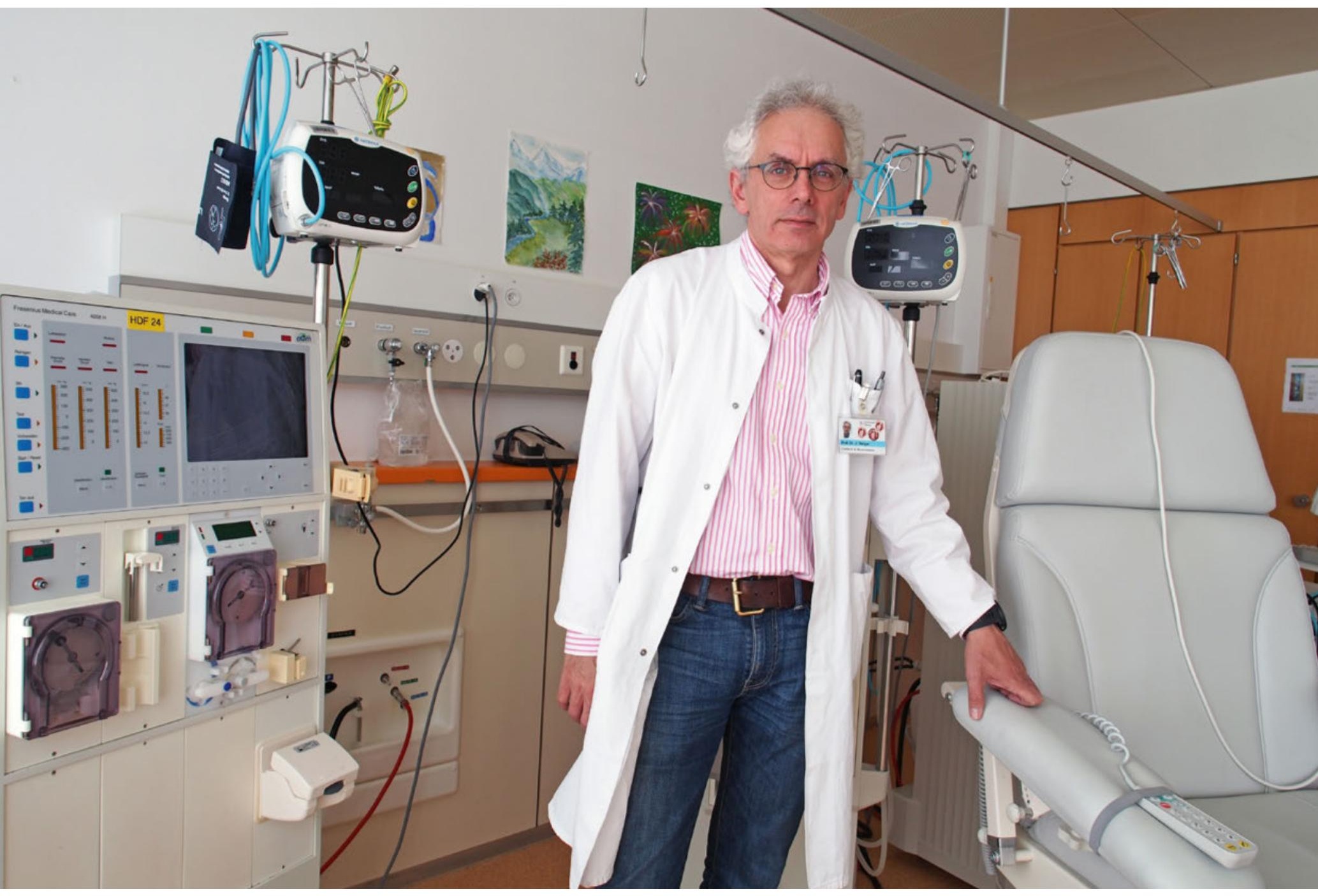


la maison: «Elles plongent notre vie de famille dans une ambiance de colocation.»

Nous faisons un petit tour, passons devant l'échographie, la salle de biopsie, la petite pièce où sont analysées les urines, avant d'arriver à la station de dialyse. Nous rencontrons des patient(e)s et des membres du personnel, échangeons blagues et rires ici ou là. Un médecinadjoint se présente comme la cheffe du chef, une grande estime mutuelle est perceptible de part et d'autre.

Quelque 11000 dialyses sont effectuées chaque année au $4^{\mathrm{e}}$ étage de la Clinique II et entre 60 et 80 transplantations sont organisées et surveillées depuis ce service. Grâce à la bonne coopération avec la chirurgie, les soins intensifs, la pathologie, l'infectiologie, la médecine et la néphrologie, le centre de transplantation de Bâle joue un rôle majeur sur le plan national et international, constate Jürg Steiger, comme en écho à sa réflexion: "une tribu soudée». Cette clinique a de quoi être fière: elle est la première en Suisse à avoir effectué une transplantation rénale à partir d'un donneur vivant, la première au monde à avoir réalisé une transplantation sur un diabétique et elle a opéré les premières transplantations dites "ABO incompatibles» en Suisse.

Mais loin de se perdre dans les hautes sphères, Jürg Steiger enchaîne sur le même ton: «En fait, en tant que néphrologue, je suis médecin de famille.» Il suit de très près la plupart de ses patient(e)s depuis longtemps, «cela crée des liens étroits, cela soude.» C'est précisément ce qu'il veut, même s'il lui faut en même temps toujours poser des limites sur le plan professionnel. "J'aime bien ce rôle de docteur", je tiens à prendre soin de mes patients. Beaucoup d'entre eux font l'expérience d'une sorte de seconde naissance après une transplantation. Oui: «Nous pouvons donner une seconde chance à beaucoup de patients.»

\section{Médecine et éthique}

La néphrologie et l'immunologie ont beaucoup de choses en commun, explique Jürg Steiger. Ce qui le fascine dans sa discipline, c'est l'ampleur de son champ: «La néphrologie s'étend de la biologie moléculaire aux questions psychologiques et éthiques en passant par les problématiques cliniques.» En tant que président de la Commission Centrale d'Ethique de l'Académie Suisse des Sciences Médicales (ASSM), il s'est récemment exprimé sur la question de savoir si le don d'organe après un suicide assisté est contraire à l'éthique ou non. «Si la décision du suicide assisté a été prise librement et en bonne et due forme et que la personne souhaite faire un don, je ne vois pas d'objections morales", déclaret-il. Mais il souligne que les directives actuelles de l'ASSM ne considèrent le suicide assisté comme accep-

\section{Jürg Steiger}

Prof. Dr méd. Jürg Steiger est né à Bâle en 1959. C'est aussi là qu'il a effectué sa scolarité et ses études de médecine. Après avoir obtenu l'examen d'Etat en 1985, il a travaillé chez Ciba-Geigy et dans différents hôpitaux de Bâle. De 1992 à 1995, il a effectué un séjour de recherche à la Harvard Medical School et au Beth Israel Hospital de Boston. Depuis 1999, il est professeur titulaire à I'Université de Bâle et, depuis 2000, médecin-chef en néphrologie et immunologie de transplantation à I'Hôpital universitaire de Bâle. Depuis 2013, il est aussi directeur du ressort Médecine et membre de la direction de l'hôpital. En 2016, il a été élu président de la Commission Centrale d'Ethique de l'Académie Suisse des Sciences Médicales. Jürg Steiger est marié et père de deux filles adultes. II vit avec sa famille à Bâle.

table du point de vue éthique que dans les cas isolés et en fin de vie. La question renvoie à une thématique plus large: «Le besoin d'organes est supérieur à l'offre. Ou en d'autres termes: la médecine de transplantation est victime de son propre succès, c'est parce qu'elle a de bons résultats que de plus en plus de patients envisagent cette option thérapeutique: «En ce qui concerne les reins, à Bâle, près de $50 \%$ sont des dons vivants. «En Finlande, ce pourcentage est nul», explique Jürg Steiger. «Parce que le responsable du centre de transplantation local considère cette pratique comme contraire à l'éthique. Pourtant, nous savons que les dons vivants donnent de meilleurs résultats que les dons cadavé-

\section{"La médecine de transplantation est victime de son propre succès.»}

riques, étant entendu que le risque pour le donneur vivant est acceptable.» En Suisse, la forme la plus fréquente de don vivant est le don entre partenaires d'un couple. "On considérait autrefois que cela n'était pas bon du point de vue psychologique», explique Jürg Steiger. "Aujourd'hui, on sait que le don entre partenaires d'un couple est psychologiquement plus prometteur que le don entre frère et sœur. Les deux partenaires en profitent, ils peuvent par exemple partir ensemble en vacances.»

Dans son travail quotidien, le médecin-chef est confronté à des questions éthiques à chaque fois qu'il y a plusieurs receveurs possibles pour un organe. Lâge d'un patient ne devrait en fait pas entrer en ligne de compte dans la décision sous peine de discrimination. «Mais imaginez que vous ayez à choisir entre une jeune femme qui élève seule ses enfants et une retraitée célibataire de 70 ans - à qui donneriez-vous l'organe? Ou encore, entre un directeur d'usine qui emploie 300 salariés, dont l'usine va fermer s'il n'obtient pas l'organe, 
et un simple mécanicien. Quelle décision prendriezvous?» Il est évident que «même si l'on a tendance, dans le premier cas, à favoriser la mère parent unique, conformément à la Constitution et à la Loi fédérale sur la transplantation d'organes, aucune critère d'ordre social ne doit entrer en ligne de compte dans l'attribution. Le système SOAS, "Swiss Organ Allocation System», aide à trancher ce genre de questions complexes: il établit une liste de points à partir de différents critères. «Un des critères est le caractère approprié de l'organe du point de vue médical mais aussi le délai d'attente déjà écoulé. Le classement établi par ce logiciel me décharge du reproche d'être injuste.»

\section{Le cœur et les reins}

Quand la langue allemande en dit long ... Que signifie l'expression "Das geht mir an die Nieren" (littéralement: "cela me porte sur les reins", équivalent de "cela me prend aux tripes») pour Jürg Steiger? Tout comme l'expression «etwas auf Herz und Nieren prüfen" (littéralement: «examiner quelque chose au niveau du cœur et des reins", équivalent de "examiner sous toutes les coutures»), cela montre à quel point le rein est important en tant qu'organe, répond le professeur de néphrologie. «Il est notre station d'épuration, il régule l'équilibre hydrique, électrolytique et acido-basique, il produit des hormones. Compte tenu de cette importance, la nature nous a donné une grande réserve. Nous utilisons seulement environ $10 \%$ de la capacité totale de nos reins.» Mais revenons à la question: «Was geht Ihnen persönlich an die Nieren?» «Lorsque ma fille revient découragée d'un concours hippique, cela me rend triste moi aussi», répond Jürg Steiger. "Si je regarde un film dramatique, il m'arrive d'avoir les larmes aux yeux. Mais le sort des patients me bouleverse également, dans le contexte professionnel, il m'arrive souvent d'être profondément touché.»

Ici, le sort d'un patient est souvent la mort imminente. Or, Jürg Steiger représente le Fonds National Suisse dans le pôle de recherche national «Fin de vie». Il a réalisé une étude de faisabilité, collaboré à esquisser les grandes lignes du programme et rédigé le projet de mise en œuvre avec le groupe de direction. «Mourir, oui ou non", c'est un thème qui m'accompagne régulièrement. Un événement clé a été le cas d'une patiente qui souhaitait mourir et demandait que l'on arrête la dialyse. Une question s'est alors imposée: es-tu en train de "jouer à Dieu»? Ma réponse a été: «non, je laisse la nature suivre son cours.»

Mais Jürg Steiger cite aussi le cas d'un patient qui a été dialysé contre son gré. «Il y avait eu un concile éthique, tout le monde était dépassé. On finit par décider qu'il n'était pas permis d'arrêter la dialyse - or le patient est décédé quand même. Un traitement imposé - une mauvaise expérience». Son grand-père lui vient à l'esprit lorsqu'il songe à un contre-exemple positif: «il est décédé d'une mort naturelle et a été exposé pendant encore un temps. Si l'on transpose cet exemple à un autre niveau: il est important qu'une personne puisse 〈partirı tranquillement.»

Vue sous cet angle, la médecine palliative fait aussi partie intégrante de sa discipline. "A mes yeux, on ne doit pas la distinguer de l'accompagnement médical qui précède, il s'agit uniquement d'un autre but thérapeutique.» Et la mort - est-ce que c'est un sujet pour Jürg Steiger? «A la maison, nous parlons souvent de ce genre de questions", dit-il. "En ce qui me concerne, je n'ai pas encore couché mes dernières volontés sur le papier. Car je constate régulièrement que les patients décident autrement lorsqu'ils sont dans une situation concrète.»

\section{Le bricoleur}

Les discussions philosophiques font partie intégrante du quotidien de Jürg Steiger. Mais il a aussi un tout autre côté: "Je fais beaucoup de choses de mes mains.» Des travaux sur bois, par exemple. "La table que nous avions à Boston, c'est moi qui l'avais fabriquée, de même que la table à langer pour nos deux filles.» Mais Jürg Steiger a aussi confectionné lui-même un gigantesque cerf-volant multicolore: «il m'a fallu presque 1000 mètres de fil.» Nous le regardons sur l'ordinateur,

\section{A qui donneriez-vous l'organe?}

il ressemble à un parapente. Photo suivante: une grande plage de sable en Bretagne. Jürg Steiger fonce sur un chariot métallique à trois roues, soudé par ses soins, dont la voile est un grand morceau de tissu qu'il a cousu lui-même. "Je confectionnais déjà mes pantalons moi-même lorsque j'étais à l'école. A mon mariage, j'ai reçu une machine à coudre en cadeau", raconte Jürg Steiger, avec le même naturel que quand il parle de transplantation rénale.

Le regard de l'intervieweur tombe sur la photo d'une prairie en fleurs sur un calendrier puis sur la chaise de bureau rouge. Jürg Steiger regarde la photo en noir et blanc de ses filles avec les vaches. Ses rêves? «Faire de l'escalade au Tibet par exemple. Voyager dans de beaux paysages. Et voir régulièrement une mer déchaînée.» Et à part cela? Sur le plan professionnel?

"Je suis satisfait de ce que je fais», dit-il. Il montre la photo clé d'une conférence personnelle qu'il a récemment donnée. On y voit: «Work hard - play hard.» 